\title{
One year of COVID-19: infection rates and symptoms in patients with inherited metabolic diseases followed by MetabERN
}

\author{
Laura Paneghetti ${ }^{1}$, Cinzia Maria Bellettato ${ }^{1}$, Annalisa Sechi ${ }^{1}$, Karolina M. Stepien² and Maurizio Scarpa ${ }^{1 *}$ (D)
}

\begin{abstract}
Background: Since the beginning of the COVID-19 pandemic, MetabERN has been monitoring the SARS-CoV-2 infection rates within its metabolic community. To gather data on the total number of cases and the severity of symptoms among IMD patients one year into the pandemic, an online survey was distributed among all MetabERN healthcare providers (HCP). Epidemiological analysis was performed by integrating the survey's data with the MetabERN database.

Results: Survey's respondents reported a total of 452 cases of COVID-19 among their IMD patients (213 paediatric and 239 adults). Considering the total number of patients followed by the respondents $(n=26,347)$, the registered prevalence of COVID-19 in the IMD population was of $1716 \times 100,000$. Italy emerged as the most affected country (25.4\% of cases), followed by the United Kingdom (14.2\% of cases). Most of the paediatric cases of COVID-19 displayed no or mild symptoms during the disease: $34 \%$ of HCP reported having asymptomatic patients in $75-100 \%$ of cases, while $37.5 \%$ reported mild symptoms in about a quarter of their patients. Similarly to paediatric cases, most adult IMD patients with COVID-19 were asymptomatic or had mild symptoms: about one third of respondents reported $75-100 \%$ asymptomatic patients and about $65 \%$ of HCP had between 0 and $50 \%$ of patients with mild symptoms. The majority of the respondents reported no deaths due to COVID-19 in adult and paediatric patients with IMDs.

Conclusions: Most of MetabERN's IMD patients who got COVID-19 during the first year of the pandemic had mild symptoms and a positive outcome of the disease. However, fatal events were recorded in paediatric patients; this, together with the lack of information on the long-term effects of COVID-19 in IMDs, call for caution in the metabolic population.
\end{abstract}

Keywords: Inherited metabolic disorders, IMDs, COVID-19, SARS-CoV-2

\section{Background}

Emerged in December 2019 in China, the Coronavirus disease 2019 (COVID-19) caused by the severe acute respiratory syndrome coronavirus 2 (SARS-CoV-2) is still a global pandemic. As of $4^{\text {th }}$ October 2021, there have been 234,809,103 confirmed cases worldwide; of these, almost

\footnotetext{
${ }^{*}$ Correspondence: maurizio.scarpa@metab.ern-net.eu

${ }^{1}$ MetabERN, Regional Coordinating Center for Rare Diseases, Udine University Hospital, Udine, Italy

Full list of author information is available at the end of the article
}

71 million have been in Europe [1]. Various vaccines to prevent COVID-19 have been approved by the European Medicines Agency [2], and their distribution among the European population is progressing at good rates, with $79 \%$ of the adult population in EU/EEA having received the first dose and $73.5 \%$ being fully vaccinated as of 4th October 2021 [3]. However, in 2021 new variants of the SARS-CoV-2 have spread and raised concerns; among these, the variant B.1.1.7 (alpha), first identified in the United Kingdom, appears to be more transmissible than previous strains and may cause more severe infection, 
while the variant B.1.617.2 (delta), detected at first in India, shows an even higher transmissibility [4]. These aspects are complicating the situation globally.

The European Reference Network for Hereditary Metabolic Disorders (MetabERN), representing 78 healthcare providers (HCP) from 23 EU Member States and 41 patients' organizations (PO), follows almost 33,000 patients with rare inherited metabolic disorders (IMD). Since the beginning of the pandemic, the network has been monitoring the SARS-CoV-2 infection rates within its metabolic community. Data gathered between March and April 2020, at the beginning of the emergency, showed that the incidence of COVID-19 in the European IMD community was of $72.9 \times 100,000$ for paediatric and adult cases, which was lower than that of the general European population (EU/EEA and UK) reported from 1st January to 7th April $2020(117 \times 100,000)$ [5]. Thanks to local restrictions, emergency protocols put into place by $\mathrm{HCP}$, and specific information shared with the patients, these data suggested that the network of $\mathrm{HCP}$ and $\mathrm{PO}$ was able to protect metabolic patients from SARS-CoV-2 infection, at least in part, during the first wave [5].

To our knowledge, at present there is no data available regarding the frequency of COVID-19 among IMD patients and the severity and consequences of the disease in metabolic patients. To gather this type of information, MetabERN distributed a dedicated survey among its members one year into the pandemic; here we present the results of the survey.

\section{Results}

Sixty metabolic experts distributed in 50 centres across 21 European countries and following a total of 26,347 IMD patients replied to our survey. Of these HCP, 11.7\% care for adult patients, $30 \%$ follow paediatric cases, while $58.3 \%$ follow both (Table 1). Each center usually cares for IMD patients with different types of conditions; the most represented in our survey were (1) lysosomal storage disorders (LSD), (2) carbohydrate, fatty acid oxidation and ketone bodies disorders (C-FAO), (3) amino and organic acids-related disorders (AOA), and (4) disorders of pyruvate metabolism, mitochondrial oxidative phosphorylation, thiamine transport and metabolism, and Krebs cycle defects (PM-MD), which are treated by $85 \%, 77 \%$, $75 \%$ and $72 \%$ of respondents, respectively (Table 1 ).

Survey participants reported a total of 452 cases of COVID-19 among their IMD patients since the beginning of the pandemic: 213 paediatric and 239 adults. Considering that total number of patients followed by the respondents $(n=26,347)$, the registered prevalence of COVID-19 in the IMD population of reference is of $1716 \times 100,000$. With a total of 115 cases $(25.4 \%)$, Italy emerged as the most affected country, followed by the United Kingdom (64 cases; 14.2\%) (Table 2 and Fig. 1).

The majority of respondents (56.7\%) have had cases of SARS-CoV-2 infection among their paediatric patients (Table 1); a high number of these were in patients affected by AOA (59.4\%), followed by LSD (40.6\%) and C-FAO (34.4\%) (Table 1). Most of the paediatric cases of COVID-19 displayed no or mild symptoms during the disease: $34 \%$ of respondents said that their patients were asymptomatic in $75-100 \%$ of cases, while $37.5 \%$ HCP reported mild symptoms in about a quarter of their patients (Fig. 2). The number of severe cases of COVID-19 was negligible: less than $10 \%$ of respondents said that $1-25 \%$ of their patients had severe symptoms or required hospitalisation (Fig. 2). Importantly, 97\% of HCP had no fatalities among their IMD patients affected with COVID-19; however, 3\% of HCP reported deaths in 1-25\% of infected patients (Fig. 2). In most of these patients, death was directly related to the viral infection, with some patients having the COVID-19 superimposed on their slowly progressing neurodegenerative condition (personal observations from clinical experience of the authors).

SARS-CoV-2 infection among adult IMD patients was reported by $44.8 \%$ of respondents (Table 1 ). The significant proportion of these cases were patients with LSD (69.2\%), AOA (46.2\%) and C-FAO (34.6\%) (Table 1). Similarly to paediatric cases, most adult IMD patients who got COVID-19 were asymptomatic or had mild symptoms: about one third of respondents reported $75-100 \%$ asymptomatic patients and about $65 \%$ of HCP had between 0 and $50 \%$ of patients with mild symptoms (Fig. 3). However, 23\% of respondents also reported about $1-25 \%$ of cases developing severe symptoms and managing either at home or requiring hospitalisation, while about $8 \%$ of HCP said that $50-75 \%$ of their COVID-19 patients were severe but were not hospitalised (Fig. 3). Compared to paediatric cases, COVID-19-related deaths in adult IMD patients were slightly higher: $11.5 \%$ of respondents said that $1-25 \%$ of their patients have died, and for almost $4 \%$ of HCP this percentage was $25-50 \%$ (Fig. 3). Nonetheless, the majority of respondents (84.6\%) report no deaths due to COVID-19 in adult patients with IMDs (Fig. 3).

\section{Discussion}

At the time the survey was concluded (end of March 2021) the ECDC reported $27,570,251$ cumulative cases of COVID-19 in the EU/EEA region since January 2020 [6], equal to a prevalence of $6094 \times 100,000$ in the general population of Europe. Compared to this rate and based on the data presented in this paper, the prevalence we recorded in MetabERN's IMD population was 
Table 1 Groups of patients and IMD types followed by MetabERN's HCP and affected by COVID-19

\begin{tabular}{lc}
\hline Category & $\mathbf{n}(\%)$ \\
\hline Group of IMD patients followed at the centre & $7 / 60(11.7)$ \\
Adult & $18 / 60(30)$ \\
Paediatric & $35 / 60(58.3)$ \\
Both & \\
IMD types that affect the patients under the respondent's care ${ }^{a}$ & $51 / 60(85)$ \\
LSD & $46 / 60(76.7)$ \\
C-FAO & $45 / 60(75)$ \\
AOA & $43 / 60(71.7)$ \\
PM-MD & $38 / 60(63.3)$ \\
CDG & $25 / 60(41.7)$ \\
NOMS & $25 / 60(41.7)$ \\
PD &
\end{tabular}

Since the beginning of the pandemic, have you had any paediatric IMD patients confirmed positive for SARS-CoV-2 by either antigen, molecular or antibody testing?

Yes

No

$22 / 60(36.7)$

Do not know

$4 / 60(6.7)$

No answer

$0 / 60(0)$

Among your paediatric patients who tested positive for COVID-19, which IMD types were mainly affected?

AOA

19/34 (55.9)

LSD

13/34 (38.2)

C-FAO

$11 / 34(32.4)$

CDG

$5 / 34(14.7)$

PM-MD

2/34 (5.9)

PD

$0 / 34(0)$

NOMS

$0 / 34(0)$

No answer

2/34 (5.9)

Since the beginning of the pandemic, have you had any adult IMD patients confirmed positive for SARS-CoV-2 by either antigen, molecular or antibody testing?

Yes

No

Do not know

No answer 2/60 (3.3)

Among your adult patients who tested positive for COVID-19, which IMD types were mainly affected? ${ }^{a}$

LSD

$18 / 26(69.2)$

$\mathrm{AOA}$

$12 / 26(46.2)$

$\mathrm{C}-\mathrm{FAO}$

9/26 (34.6)

PM-MD

4/26 (15.4)

PD

$1 / 26(3.9)$

CDG

$1 / 26(3.9)$

NOMS

1/26 (3.9)

No answer

0/26 (0)

$A O A$ amino and organic acids-related disorders, $P M-M D$ disorder of pyruvate metabolism, Krebs cycle defects, mitochondrial oxidative phosphorylation disorders, disorders of thiamine transport and metabolism, C-FAO carbohydrate, fatty acid oxidation and ketone bodies disorders, LSD lysosomal storage disorders, $P D$ peroxisomal disorders, CDG congenital disorders of glycosylation and disorders of intracellular trafficking, NOMS disorders of neuromodulators and other small molecules

${ }^{a}$ More than one answer possible 
Table 2 Number of COVID-19 cases reported by MetabERN's HCP

\begin{tabular}{|c|c|c|c|c|c|c|}
\hline Country & Paediatric & Adult & Total & $\%$ & Responding centres ${ }^{a}$ & $\begin{array}{l}\text { Tot number of } \\
\text { centres }{ }^{\mathrm{a}} / \text { country }\end{array}$ \\
\hline Italy & 59 & 56 & 115 & 25.4 & 8 & 11 \\
\hline UK & 2 & 62 & 64 & 14.2 & 2 & 6 \\
\hline France & 26 & 28 & 54 & 11.9 & 5 & 9 \\
\hline Netherlands & 35 & 16 & 51 & 11.3 & 3 & 5 \\
\hline Spain & 13 & 27 & 40 & 8.8 & 3 & 5 \\
\hline Belgium & 14 & 16 & 30 & 6.6 & 5 & 6 \\
\hline Germany & 23 & 7 & 30 & 6.6 & 6 & 9 \\
\hline Portugal & 19 & 6 & 25 & 5.5 & 4 & 5 \\
\hline Denmark & 10 & 5 & 15 & 3.3 & 1 & 1 \\
\hline Slovakia & 2 & 6 & 8 & 1.8 & 1 & 1 \\
\hline Poland & 0 & 6 & 6 & 1.3 & 1 & 1 \\
\hline Slovenia & 5 & 0 & 5 & 1.1 & 1 & 1 \\
\hline Croatia & 3 & 0 & 3 & 0.7 & 1 & 1 \\
\hline Latvia & 0 & 3 & 3 & 0.7 & 1 & 1 \\
\hline Hungary & 1 & 1 & 2 & 0.4 & 1 & 1 \\
\hline Bulgaria & 1 & 0 & 1 & 0.2 & 1 & 1 \\
\hline Austria & 0 & 0 & 0 & 0.0 & 1 & 2 \\
\hline Lithuania & 0 & 0 & 0 & 0.0 & 1 & 1 \\
\hline Malta & 0 & 0 & 0 & 0.0 & 1 & 1 \\
\hline Norway & 0 & 0 & 0 & 0.0 & 3 & 2 \\
\hline Sweden & 0 & 0 & 0 & 0.0 & 1 & 2 \\
\hline Total & 213 & 239 & 452 & $100 \%$ & 50 & 72 \\
\hline
\end{tabular}

${ }^{a}$ Centres belonging to the MetabERN network

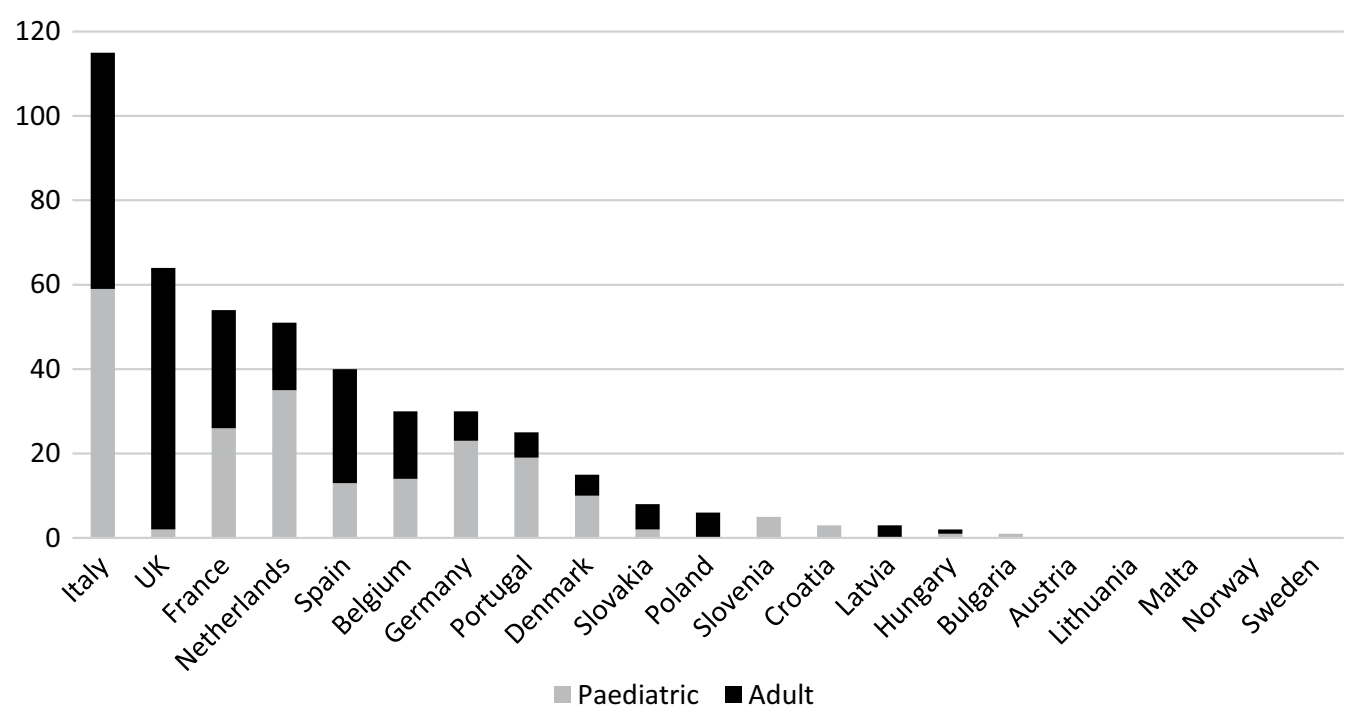

Fig. 1 COVID-19 cases among MetabERN's IMD patients per country

much lower $(1716 \times 100,000)$. This observation may be due to the fact that IMD patients' and their caregivers' perception of being at risk of COVID-19-related complications probably prompt them to be keener than the general population in respecting preventative measures like social distancing, increased hand 

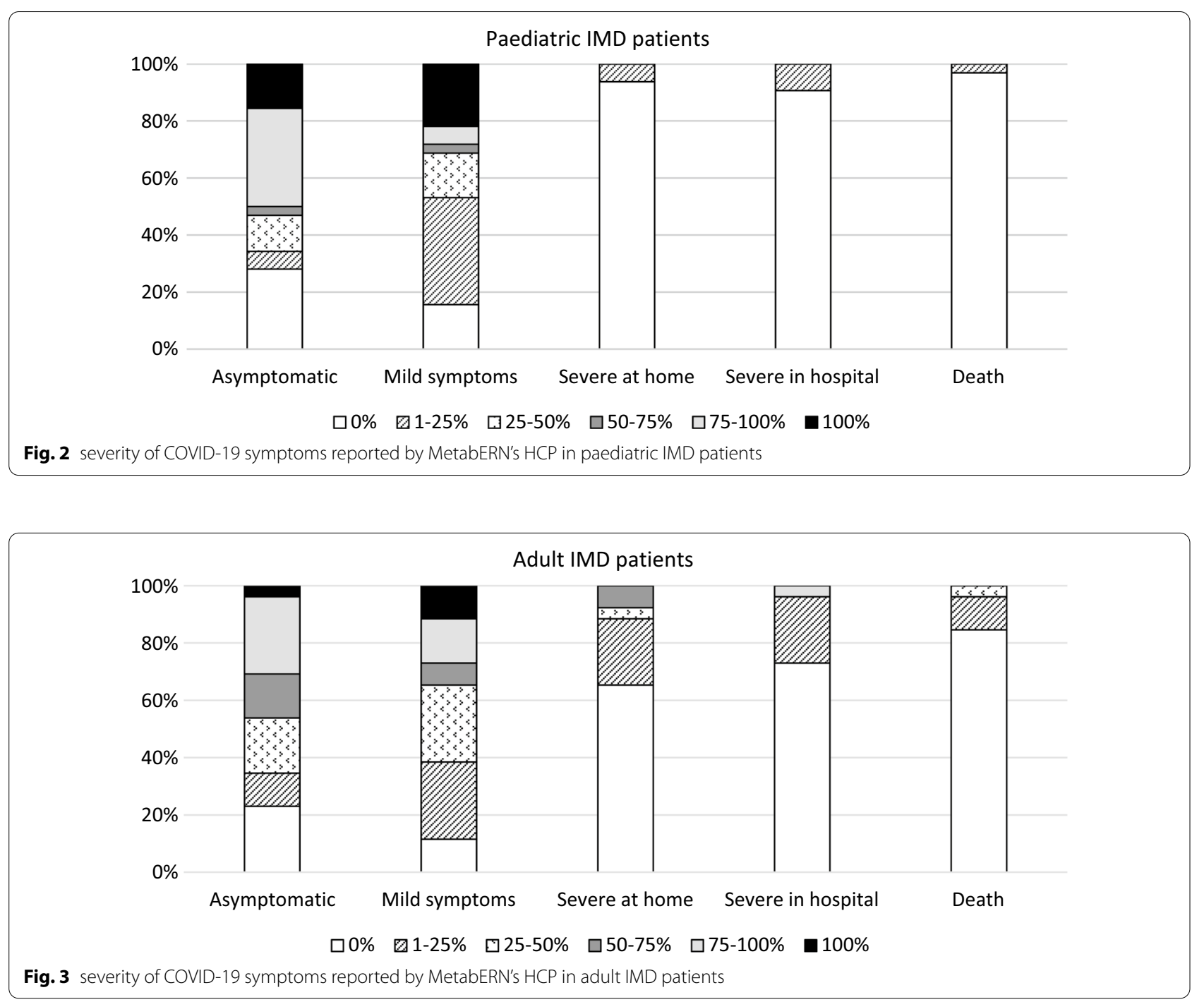

washing, mask wearing, and decreased social interactions and activities [7, 8]. As a result, patients with IMD continued to shield throughout most part of 2020 and 2021. Moreover, as mentioned in a previous publication [5], the low prevalence of COVID-19 among IMD patients may be the result of a solid network of HCPs and patient organizations that worked together to effectively protect metabolic patients from SARSCoV-2 infection by implementing telemedicine tools and home therapy, where available, or by shifting the workload to the primary care setting [9]. Lastly, this could also be the result of MetabERN's recommendations on COVID-19, which have been largely and effectively disseminated inside the network [10]

The country that reported most cases of COVID-19 among IMD patients was Italy. This may be due to the fact that Italy is the EU country with one of the highest rates of COVID-19 infections; another reason for this result is that Italy was the country that participated the most to the survey (eight centres in total).

In our survey, most paediatric and adult IMD patients affected with COVID-19 were known to have an underlying metabolic disorder such as AOA, LSD, or C-FAO. These disorders are generally considered at high risk of metabolic decompensation, cardio-respiratory complications, and frequent exacerbation induced by infections, so they may be more vulnerable also to COVID-19-induced complications [8]. Most of the survey respondents are aware that viral infection may instigate acute metabolic decompensation, which results in Intensive Care Unit admission, requires prolonged in-hospital stay, and often haemofiltration and the use of intravenous medications. The prognosis of such complex cases with several risk factors can be poor. However, we cannot say for certain that patients with AOA, LSD, or C-FAO have a higher risk of severe acute metabolic illness or other 
viral-related complications when infected with SARSCoV-2. Indeed, according to our data, most IMD patients with COVID-19 (both paediatric and adults) had no or mild symptoms.

In line with the general population and compared to the paediatric patients [11], adults with an IMD who contracted SARS-CoV-2 infection experienced more severe symptoms, required hospitalisation or died from COVID-19-related complications. Based on the available evidence, we cannot say that compared to the general population IMD patients were more "protected" from COVID-19, because they contracted the infection and there were some cases that resulted in death, even among paediatric cases. At the same time, at this stage we do not have enough data to say whether IMD patients are at a higher, lower or equal risk of COVID-19-related complications compared to the general population; more studies are needed to clarify this aspect.

Since the pathway of SARS-CoV-2 infection involves lysosomes trafficking for egress [12], some authors have hypothesized that LSDs such as Niemann Pick type C and Gaucher disease are less at risk of SARS-CoV-2 infection or severe COVID-19 complications [13, 14]. Our data demonstrate that LSDs are not immune towards COVID19; on the contrary they were the largest group of the metabolic disorders in our adult casualty list. While this result is probably due to the fact that most of the survey respondents are specialised in LSDs and follow this type of patients, due to the limitations of the survey we cannot add any further comment regarding the specific LSD conditions.

This study has several limitations. Firstly, the data presented is limited to 50 out of 78 centres belonging to the MetabERN network, so we cannot draw conclusions regarding the entire MetabERN network or the overall IMD population in Europe and around the world. Secondly, the reported number of affected patients is certainly underestimated (low case detection) as not all centers in the MetabERN network contributed with their data. In addition, not all IMD patients followed by the network were tested for COVID-19: only symptomatic cases, those with a family member affected with COVID19 or those that require investigations such as a pulmonary function test are usually tested; also, it is possible that some patients were affected with a mild infection that did not require hospitalization and felt like it was not necessary for them to report it to their metabolic physician. Furthermore, IMD patients who developed complications of COVID-19 might have had other comorbidities such as diabetes mellitus, increased body mass index, or were of certain ethnic origin (BAME). These factors might have impacted the course of their illness and their recovery from it. The detailed information regarding the above was not collected in the survey. Lastly, in MetabERN's centers there is a high representation of LSD patients, which does not accurately reflect the reality of IMD patients in Europe. Given the vast differences in local epidemiology and policy interventions and the uneven geographical distribution of MetabERN HCP across each country and across the EU, more time and data are needed to determine the actual consequences of the COVID-19 pandemic on the IMD population.

Many changes in hospital visits and disease management were implemented at the very beginning of the pandemic to protect patients, especially because experts thought that IMD patients were at a higher risk to develop severe forms of COVID-19 [5]. In April 2020, MetabERN published its recommendations for all IMD patients and caregivers about treatment adherence during the emergency [8]. In March 2021, the network published additional recommendations for vaccination based on the available data, which suggested that infections could start a metabolic decompensation in IMDs such as AOA, PM-D, C-FAO or LSD, and put patients at risk of metabolic decompensation, respiratory or cardiac complications, and frequent exacerbation [8]. The vaccination among the paediatric population is still a matter of debate worldwide. The fatal events in some paediatric patients reported in our study prompt MetabERN to recommend the inclusion of paediatric IMD patients as a priority group for COVID-19 vaccination. There is still a lot unknown about the long-term effects of COVID19 in the general population and especially in metabolic patients. These survey results warrant further research into the impact of COVID-19 on the IMD population.

\section{Conclusions}

During the first year of COVID-19 pandemic, the majority of MetabERN's IMD patients who got the disease experienced mild symptoms and had a positive outcome. However, the fatal events recorded among paediatric patients together with the limited knowledge regarding the long-term effects of COVID-19 call for caution and special attention in the metabolic population. More time is needed to perform further studies on the topic; in the meantime, IMD paediatric patients should be included as a priority group for COVID-19 vaccination.

\section{Methods}

The Survey Monkey platform was used to design and distribute the survey and collect data. The survey included 12 questions, was distributed to all MetabERN centers and was active between 20 and 29th March 2021 (Additional file 1). The data were extracted and analysed with Microsoft Excel. Epidemiological analysis was performed by integrating the survey's data with the MetabERN 
database, which includes the number of adult and paediatric patients followed by each HCP member of the network, where a patient is considered an adult when he/ she reaches the age of 18 years. For the statistical analysis, the total number of patients followed by the centres that responded to the survey $(n=26,347)$ was used as the population of reference.

\begin{abstract}
Abbreviations
IMDs: Inherited metabolic disorders; HCP: Healthcare provider; MetabERN: European Reference Network for Rare Hereditary Metabolic Disorders; AOA: Amino and organic acids-related disorders; PM-MD: Disorder of pyruvate metabolism, Krebs cycle defects, mitochondrial oxidative phosphorylation disorders, disorders of thiamine transport and metabolism; C-FAO: Carbohydrate, fatty acid oxidation and ketone bodies disorders; LSD: Lysosomal storage disorders; PD: Peroxisomal disorders; CDG: Congenital disorders of glycosylation and disorders of intracellular trafficking; NOMS: Disorders of neuromodulators and other small molecules.
\end{abstract}

\section{Supplementary Information}

The online version contains supplementary material available at https://doi. org/10.1186/s13023-022-02247-3.

Additional file 1. Original survey questionnaire

\section{Acknowledgements}

This work was generated within the European Reference Network for Rare Hereditary Metabolic Disorders (MetabERN). The authors would like to thank Dr. Adrian Haslam for his help with the data analysis and the survey design. The authors are also grateful to all MetabERN members who replied to the survey: A. Hlavata, A.M. Bosch, A. van der Ploeg, Aasne Aarsand, A. Garcia, Alain Fouilhoux, Albert Hagege, Alberto Burlina, Alberto Piperno, Alessandro Simonati, Aline Cano, Allan Lund, A.M. Pocubayova, A.N. Jonckheere, Ana M. Gaspar, Andrea Bordugo, Andreas Hahn, Anupam Chakrapani, Aurelie Hubert, Balogh, Barbara Plecko, B. Chabrol, Benedicte Heron, Bernassola Aline, Birute Tumiene, Bruno Frediani, Carlo Dionisi-Vici, Carmo Macario, Charlotte Dawson, Christina Lampe, Claudia S. Costa, Corinne Delaet, D. Ramadza, Daniela Karall, Das Anibh, Dominique Germain, Dominique Roland, Doriette M. Soler, Dorothea Moeslinger, Dries Dobbelaere, Dulce Quelhas, E. Leaoteles, Efstathia Chronopoulou, Eliane Sardh, Elisabeth Jameson, Enrico Bertini, Esmeralda G. Martins, Esmeralda Rodrigues, Estela Rubio, Eva Morava, Francois Eyskens, F.J. van Spronsen, Florian Lagler, Ferruccio Santini, F.G. Debray, Flavia Tubili, Frank Rutsch, Freddy Raymackers, G. Visser, Germaine Pierre, Gert Matthijs, Giovanni Ceccarini, Giulia Polo, Giuseppe Banderali, Graziella Cefalo, H. Huidekoper, Herve Puy, Illsinger Sabine, I. Tournev, Ivan Falzon, Ivo Baric, James Clark, James Davison, Jen-Baptiste Arnoux, Jord Perez, Jorge Asin Cayela, Julia Hennermann, Karine Mention, Karl-Eugen Mengel, K. Kwaskowska, Klaus Mohnike, K. Koczok, Leticia Ceberiohualde, Lise Aksglaede, Liz Morris, Luis Jose Aldamiz, M. Engelen, Madara Masinska, Maja Di Rocco, Malecki, Manuel Schiff, Maria Donati, Maria Luz Couce Pico, Merie-Cecile Nassogne, Marie-Francois Vincent, Mark Gruppetta, Marquat, Martin Zenker, M.B. Kiec, M.C.G.J. Brouwers, Maria Del Toro, M. Garcia Silva, Michelle Grzybowski, Miguel F. Gago, Mojca ZerjavTansek, Montserrat Morales, Muschol, N. Verhoeven, Nadia Belmatoug, Natalie Weinhold, Nathalie Guffon-Fouilhoux, Nicola Acquilina, Niklas Darin, Olga Zevedo, Giancarlo Parenti, Pascale De Lonlay, Patrick Deegan, Patrick Verloo, Paula Sanchez Pintos, Peter Witters, Philippe Labrune, Pietro Strisciuglio, R. Artuch, Simona Gasperini, Saikat Santra, Salvatore Grosso, Samia Pichard, arah Gruenert, Scalais Emmanuel, Simon Jones, Spyros Batzios, Stefan Koelker, Stephen Attard, Suresh Vijay, Susanne Greber-Platzer, Sverre Sandberg, Svetlana Lajic, Terry G.J. Derks, Tarekegn Hiwot, Teodoratch, Thomas Mueller, Thomas Opladen, Urh Groselj, Ute Spiekerkoetter, Valts Abols, Viktor Kozich, W. Sperl, YT Bliksrub.

\section{Authors' contributions}

LP prepared and distributed the survey, analysed the data, wrote and revised the manuscript. CMB prepared and distributed the survey, analysed the data, and revised the manuscript. AS revised the data and the manuscript. KMS revised the survey and the manuscript. MS conceived the study and revised the manuscript. All authors read and approved the final manuscript.

\section{Funding}

This work was co-funded by the European Union within the framework of the Third Health Programme ERN-2016—Framework Partnership Agreement 2017-2021, Project ID No. 739543.

\section{Availability of data and materials}

The datasets used and/or analysed during the current study are available from the corresponding author on reasonable request.

\section{Declarations}

\section{Ethics approval and consent to participate}

All surveys' participants gave their consent for data collection and publication.

\section{Consent for publication}

Not applicable.

\section{Competing interests}

The authors declare that they have no competing interests.

\section{Author details}

${ }^{1}$ MetabERN, Regional Coordinating Center for Rare Diseases, Udine University Hospital, Udine, Italy. ${ }^{2}$ Adult Inherited Metabolic Diseases, Salford Royal NHS Foundation Trust, Salford, UK.

Received: 8 October 2021 Accepted: 13 February 2022

Published online: 04 March 2022

\section{References}

1. World Health Organization. WHO Coronavirus Disease (COVID-19) dashboard 2020. https://covid19.who.int/. Accessed 20 Dec 2021.

2. European Medicines Agency. COVID-19 vaccines: authorised 2021. https://www.ema.europa.eu/en/human-regulatory/overview/publichealth-threats/coronavirus-disease-covid-19/treatments-vaccines/vacci nes-covid-19/covid-19-vaccines-authorised\#authorised-covid-19-vacci nes-section. Accessed 20 Dec 2021.

3. European Centre for Disease Prevention and Control. COVID-19 vaccine tracker 2021. https:/vaccinetracker.ecdc.europa.eu/public/extensions/ COVID-19/vaccine-tracker.html\#uptake-tab. Accessed 20 Dec 2021.

4. European Centre for Disease Prevention and Control. SARS-CoV-2 variants of concern as of 24 June 2021. https://www.ecdc.europa.eu/en/covid-19/ variants-concern. Accessed 20 Dec 2021.

5. Lampe C, Dionisi-Vici C, Bellettato CM, Paneghetti L, van Lingen C, Bond S, Brown C, Finglas A, Francisco R, Sestini S, Heard JM, Scarpa M, MetabERN collaboration group. The impact of COVID-19 on rare metabolic patients and healthcare providers: results from two MetabERN surveys. Orphanet J Rare Dis. 2020;15(2):341.

6. European Centre for Disease Prevention and Control. COVID-19 situation dashboard 2021. https://qap.ecdc.europa.eu/public/extensions/COVID19/COVID-19.html\#global-overview-tab. Accessed 20 Dec 2021.

7. Sechi A, Macor D, Valent S, Da Riol RM, Zanatta M, Spinelli A, Bianchi K, Bertossi N, Dardis A, Valent F, Scarpa M. Impact of COVID-19 related healthcare crisis on treatments for patients with lysosomal storage disorders, the first Italian experience. Mol Genet Metab. 2020;130:170-1.

8. Ryan E, Lopez G, Balwani M, Barbouth D, Burrow TA, Ginns E, Goker-Alpan O, Grabowski G, Kartha R, Kishnani P, Lau H, Lee C, Mistry P, Maegawa G, Packman S, Prada C, Rosenbloom B, Roshan Lal T, Schiffmann R, Weinreb N, Sidransky E. COVID-19 patient impact: a survey of the Gaucher community involving patients, caregivers and family members based in the US to determine impact of the pandemic. Mol Genet Metab Rep. 2021;132(2):S93. 
9. Haslam A, Evans W, Hendriksz CJ, Stepien KM. COVID: accelerating the shift of work from secondary into primary care, and on rare diseases. $\mathrm{Br} J$ Gen Pract. 2020.

10. MetabERN. COVID-19 resources 2020. https://metab.ern-net.eu/covid19/. Accessed 20 Dec 2021.

11. Badal S, Thapa Bajgain K, Badal S, Thapa R, Bajgain BB, Santana MJ. Prevalence, clinical characteristics, and outcomes of pediatric COVID-19: a systematic review and meta-analysis. J Clin Virol. 2021;135:104715.

12. Ghosh S, Dellibovi-Ragheb TA, Kerviel A, Pak E, Qiu Q, Fisher M, Takvorian PM, Bleck C, Hsu VW, Fehr AR, Perlman S, Achar SR, Straus MR, Whittaker GR, de Haan CAM, Kehrl J, Altan-Bonnet G, Altan-Bonnet N. $\beta$-Coronaviruses use lysosomes for egress instead of the biosynthetic secretory pathway. Cell. 2020;183(6):1520-35.

13. Ballout RA, Sviridov D, Bukrinsky MI, Remaley AT. The lysosome: a potential juncture between SARS-CoV-2 infectivity and Niemann-Pick disease type C, with therapeutic implications. FASEB. 2020;34(6):7253-64.

14. Ginns El, Ryan E, Sidransky E. Gaucher disease in the COVID-19 pandemic environment: the good, the bad and the unknown. Mol Genet Metab Rep. 2021;132(4):213-4.

\section{Publisher's Note}

Springer Nature remains neutral with regard to jurisdictional claims in published maps and institutional affiliations.

- fast, convenient online submission

- thorough peer review by experienced researchers in your field

- rapid publication on acceptance

- support for research data, including large and complex data types

- gold Open Access which fosters wider collaboration and increased citations

- maximum visibility for your research: over 100M website views per year

At BMC, research is always in progress.

Learn more biomedcentral.com/submissions 